Cinémas

Revue d'études cinématographiques

Journal of Film Studies

\title{
Entre le corps évanescent et le corps supplicié : Videodrome et les fantaisies postmodernes
}

\section{Vicente Sánchez-Biosca}

Volume 7, numéro 1-2, automne 1996

La Représentation du corps au cinéma

URI : https://id.erudit.org/iderudit/1000933ar

DOI : https://doi.org/10.7202/1000933ar

Aller au sommaire du numéro

Éditeur(s)

Cinémas

ISSN

1181-6945 (imprimé)

1705-6500 (numérique)

Découvrir la revue

Citer cet article

Sánchez-Biosca, V. (1996). Entre le corps évanescent et le corps supplicié :

Videodrome et les fantaisies postmodernes. Cinémas, 7(1-2), 73-88.

https://doi.org/10.7202/1000933ar

\section{Résumé de l'article}

Il est une dualité imaginaire qui domine la représentation du corps dans les discours audiovisuels de ces derniers temps. D'une part, ce que l'on pourrait appeler la " décorporéisation ", comme perte subtile d'une évocation quelconque de la chair. C'est l'imagerie laissant sa marque indélébile sur la publicité, les corps des stars classiques du cinéma hollywoodien et, plus récemment, les modèles sportifs de notre époque, dont Arnold Schwartzenegger en est le paradigme. D'autre part (et ce n'est pas sans rapport avec ce qui précède), la figuration de certains corps charnels, d'une obscénité débordante, soumis à la violence ou à la jouissance d'autrui. C'est l'imagerie qui est à la base des films de tueurs en série, ainsi que de la pornographie et dont la convergence produira le snuff. La particularité d'un film comme Videodrome (David Cronenberg, 1982) consiste en ce qu'il participe des deux aspects évoqués, tout en développant leurs rapports à l'intérieur du récit. Le but de cet article est d'analyser la dualité des corps selon les manifestations discursives qu'elle produit sur le récit et sur le point de vue adopté par celui-ci. 


\title{
Entre le corps évanescent et le corps supplicié: Videodrome et les fantaisies postmodernes ${ }^{1}$
}

\section{Vicente Sánchez-Biosca}

\begin{abstract}
RÉSUMÉ
Il est une dualité imaginaire qui domine la représentation du corps dans les discours audiovisuels de ces derniers temps. D'une part, ce que l'on pourrait appeler la "décorporéisation", comme perte subtile d'une évocation quelconque de la chair. C'est l'imagerie laissant sa marque indélébile sur la publicité, les corps des stars classiques du cinéma hollywoodien et, plus récemment, les modèles sportifs de notre époque, dont Arnold Schwartzenegger en est le paradigme. D'autre part (et ce n'est pas sans rapport avec ce qui précède), la figuration de certains corps charnels, d'une obscénité débordante, soumis à la violence ou à la jouissance d'autrui. C'est l'imagerie qui est à la base des films de tueurs en série, ainsi que de la pornographie et dont la convergence produira le snuff. La particularité d'un film comme Videodrome (David Cronenberg, 1982) consiste en ce qu'il participe des deux aspects évoqués, tout en développant leurs rapports à l'intérieur du récit. Le but de cet article est d'analyser la dualité des corps selon les manifestations discursives qu'elle produit sur le récit et sur le point de vue adopté par celui-ci.
\end{abstract}

\section{ABSTRACT}

There is an imaginative dualism that has dominated the representation of the body in the audiovisual discourses of recent times. On the one hand, there is what 
might be called "decorporalization," a subtle loss of any evocation of the flesh. This is the imagery that has left an indelible mark on advertising, on the bodies of the classic stars of Hollywood, and more recently on sports heroes, with Arnold Schwartzenegger as the paradigmatic example. On the other hand, and not unrelated to the preceding, there is a figuration of certain carnal bodies, an unbridled obscenity subject to the violence or the pleasure of the other. This is the imagery on which films about serial killers are based, as well as pornography and the convergence of the two in the snuff film. The particularity of a film like Videodrome (David Cronenberg, 1974) is the way it draws on both these aspects, while exploring the relationships between them in the unfolding of the plot. The aim of this article is to analyze this duality of bodies through its discursive manifestations in the plot, and in the point of view adopted by the plot.

\section{Une culture schizoïde}

On a souvent remarqué (et une observation empirique permet de le constater aisément) que notre époque manifeste des traits schizoïdes dans sa représentation du corps. D’un côté, en effet, nous sommes interpellés par des corps idéaux incarnés dans des images de plus en plus parfaites issues des progrès de la chirurgie plastique, de la généralisation des programmes diététiques et de la multiplication des sciences médicales appliquées fabriquant des prothèses sans défaut. Ce corps naissant, à la croisée de l'essor extraordinaire de la mode, des pratiques thérapeutiques et de la chirurgie, trouve un mode d'expression particulièrement adéquat dans la publicité télévisuelle. Par ailleurs, nous sommes confrontés à des manifestations visuelles perverses de corps subissant des marques de violence, ou bien de corps excessivement charnels, et qui se déploient dans de nombreux domaines de la culture de masses: l'information, le reportage, l'image pornographique, voire le mythique snuff. Ces corps sont morcelés et souffrants; leur humanité devient problématique, pour ne pas dire perdue. La violence dont ils sont les victimes, faut-il le rappeler, est adressée à l'œil, celui-ci étant censé dévorer avec fascination son objet. On le voit, il est question ici de 
corps extrêmes, bien que dans un sens totalement différent de celui proposé par Patrick Baudry ${ }^{2}$. Et ce n'est pas un hasard si cette attitude à l'égard du corps souffrant se trouve amplement exprimée par les films d'horreur dès les années soixante-dix (mutilations, déchirements des tissus, meurtres sauvages, images de boucherie).

Admettons qu'une telle dualité ne soit pas surprenante et, qui plus est, ne nous appartienne pas en propre. À vrai dire, nul n'ignore que toute civilisation vit dans une tension permanente entre ses rêveries et la réalité. Par conséquent, on peut difficilement en rester là et il devient alors nécessaire de déterminer le rapport existant entre ces deux notions qui s'enchevêtrent, chacune ne se définissant que par rapport à l'autre. Bien entendu, la condition sine qua non de tout idéal est de produire une tension imaginaire avec la réalité des corps. Cette tension est actuellement mise de l'avant par les promesses de "correction" des corps réels par la science moderne (médecine, biologie, généti$\left.q^{3} \mathrm{e}^{3}\right)$. Nonobstant, il est de ce fait difficile d'approfondir cette relation sans examiner de plus près les concepts utilisés et, notamment, le concept d'idéal. Nous devons donc nous poser les questions suivantes: comment construit-on un idéal du corps et, ensuite, quelles sont les caractéristiques du corps idéal postmoderne?

\section{Des corps idéaux}

Idéal. Ce terme issu de la psychanalyse fait problème. Au départ, Freud aborda ce concept sous le nom de Ich-ideal, le définissant comme une instance surgissant de la convergence du narcissisme et de l'identification que ce soit aux parents, à leurs substituts ou aux idéaux collectifs. C'est ainsi que le sujet tend vers l'idéal afin de s'y conformer lui-même dans un processus psychique en vertu duquel les qualités de l'objet sont portées à leur perfection (idéalisation). Jacques Lacan, suite à une distinction parfaitement opératoire entre idéal du Moi et Moi idéal, affirme: "L'idéal du Moi commande le jeu de relations d'où dépend toute relation à autrui. Et de cette relation à autrui dépend le caractère plus ou moins satisfaisant de la structuration imaginaire" (p. 161). Lieu de reconnaissance du sujet, l’idéal 
engendre une tension avec la réalité. Or, la formation de l'idéal étant liée au narcissisme, elle implique l'existence du refoulement ${ }^{4}$.

Compte tenu des objectifs posés par notre texte, nous ne retiendrons de cette description que deux idées. Tout d'abord, la formation d'un idéal suppose que celui-ci ne soit pas simplement imposé par quelqu'un, fût-ce ce "Pouvoir" avec un "P " majuscule dont on parlait encore récemment. Il doit au contraire être intériorisé par le sujet qui le structure et qui peut même produire des effets imaginaires sur une collectivité déterminée. Ensuite, l'idéal naît d'une identification du Moi, en dépit des croyances de l'individu (même si celles-ci ne sont pas indifférentes). On peut répondre ici à notre deuxième questionnement portant sur les caractéristiques que possède, selon nous, l'idéal corporel prévalant dans nos sociétés occidentales's. Nous en comptons quatre: le corps doit être léger (diététique), sportif (performant), ductile (changeable) et hygiénique (transparent d'un point de vue social). On reconnaîtra ici l'héritage, sous sa forme extrême, du projet du XVIII ${ }^{e}$ siècle dont Michel Foucault a parcouru les avatars dans nombre de ses livres, à savoir: dans la constitution de la médecine moderne (Naissance de la clinique), dans l'élaboration d'une science sexuelle déployant un savoir qui se veut exhaustif sur le sexe (Histoire de la sexualite), dans le développement de mécanismes de dressage du corps tels qu'ils apparaissent lorsque l'Occident prononce son dernier adieu à la torture (Surveiller et punir). Quelle que soit la correspondance entre les traits précédemment énumérés et le projet discursif classique, il est important de remarquer l'effondrement de l'équilibre atteint par le discours classique. Il n'est pas exagéré de dire que son dessein dominant semble être de se débarrasser du corps, de sa matérialité, de le rendre, si l'on préfere, transparent. Et cependant, cet évanouissement du corps entraîne un coût: la réapparition obscène de la matérialité exclue. Là où le discours classique sur le corps était unidirectionnel, l'attitude postmoderne (ou l'état postmoderne) se heurte à la conscience de l'échec.

Soyons plus précis. Comment perçoit-on la conscience de l'épuisement historique du projet classique tout en convenant que le paradigme n'a pas disparu? Pour répondre à cette ques- 
tion, on nous permettra un petit retour sur la tension constitutive de l'idéal. Nous l'avons dit, comme la tension que génère l'idéal est constitutive de sa structure, toute période vit en contradiction avec les idéaux qu'elle requiert pour subsister, et cela, aussi longtemps qu'ils demeurent des formations irréalisables. Toutefois, la particularité de notre soi-disant postmodernité est qu'une exigence d'autant plus sévère d'assujettissement à l'idéal entraîne son radical effondrement et ce, pour deux raisons. La première est liée à la désacralisation provoquée par les découvertes spectaculaires de la science qui suggère maintenant que l'atteinte de l'idéal n'est plus illusoire - ce qu'elle semblait être auparavant -, mais accessible, voire à la portée de tous. D'où un affaiblissement extraordinaire des compensations symboliques ${ }^{6}$ et, corrélativement, la soumission de l'individu à une série inépuisable et virtuellement infinie de manipulations scientifiques, génétiques, thérapeutiques, diététiques ou autres. Les implications de l'introduction de la technologie dans le domaine symbolique de la maternité constitue un bel exemple d'un trajet où les compensations ont disparu et où l'avancée de la science ne semble plus pouvoir s'arrêter ${ }^{7}$. L'expression vindicative "Mon corps est à moi » traduit fidèlement la faillite de toute transcendence et la responsabilité ultime du sujet par rapport à l'usage de son corps, à la condition cependant que ce corps qu'on proclame notre bien le plus précieux devienne patrimoine exclusif des spécialistes qui nous garantiront - dit-on - l'accomplissement de l'idéal. La deuxième raison vient des conséquences insupportables que l'échec, dans cet effort et cette soumission, implique pour le sujet. Car aucun mécanisme de deuil (symbolique donc) ne vient tempérer l'angoisse produite par cet échec. Nous assistons à une course effrénée dont la solution est sans cesse repoussée, demeurant ainsi entre les mains des scientifiques ${ }^{8}$. Ces conséquences sont à la base d'une génération d'effets pervers, de fantaisies destructives du corps dont certaines maladies d'autodestruction liées à la perception par le sujet de l'image de son corps (notamment, l'anorexie et la boulimie). En d'autres termes, plus l'idéal s'avère être exigeant, plus le corps matériel devient pesant faute de pouvoir s'y conformer. Alors, plus grande est l'angoisse s'emparant du sujet. 
Notre hypothèse pourrait être formulée comme la crise du projet corporel moderne (médical, rentable, protéiforme et performant), entrainant une déstabilisation sans précédent dans l'histoire et ce, dans plusieurs domaines: décalage entre principes juridiques et possibilités expérimentales, divorce entre science et éthique ${ }^{9}$, déchirement entre idéaux et perversions... Néanmoins, ce qui revêt pour nous, en tant que critiques de la culture visuelle, un sens encore plus décisif, c'est le déchaînement de toute une série de fantaisies sadiques qui trouvent dans l'image et dans ses fictions (le cinéma en particulier) un support d'expression privilégié. Il y a deux raisons à cela: la première est que l'image n'est pas une manifestation quelconque, mais bien plutôt le lieu d'ancrage du narcissisme fondant l'ordre imaginaire qui régit tout système d'identifications; la seconde vient du fait que notre civilisation étant une civilisation du regard, les représentations visuelles du corps deviennent un symptôme des avatars de la pulsion scopique dans le monde contemporain. Voilà pourquoi l'étude des fictions cinématographiques est susceptible de contribuer au dressage d'un bilan, fût-il conjectural, de la culture qui nous enveloppe ${ }^{10}$.

\section{Videodrome et ses deux fantaisies}

De nombreuses fictions cinématographiques illustrent l'une ou l'autre des deux tendances précédentes: la première est l'essor d'un nouveau star system du corps mécanique ou cyborg caractérisé par l'absence de différence sexuelle; tandis que la seconde se situe au cœur des images de morcèlement des corps qui peuplent les films mettant en scène des psychopathes ". Quoi qu'il en soit, si certaines de ces fictions choisissent de représenter une de ces fantaisies, rares sont celles qui tentent de les articuler de façon systématique. Videodrome (David Cronenberg, 1982) fait partie de celles-ci. Le premier mérite de ce film, à tout le moins dans notre perspective, vient de ce qu'il approfondit, au niveau diégétique, les rapports existant entre ces deux tendances postmodernes opposées. Cependant, le film ne prend pas en charge le corps idéal en tant que corps parfait et ductile, proposant plutôt une fantaisie extrême de décorporéisation conduisant à la disparition de toute chair, en une sorte d'esthétique de la disparition du corps, pour reprendre les termes de Paul Virilio. 


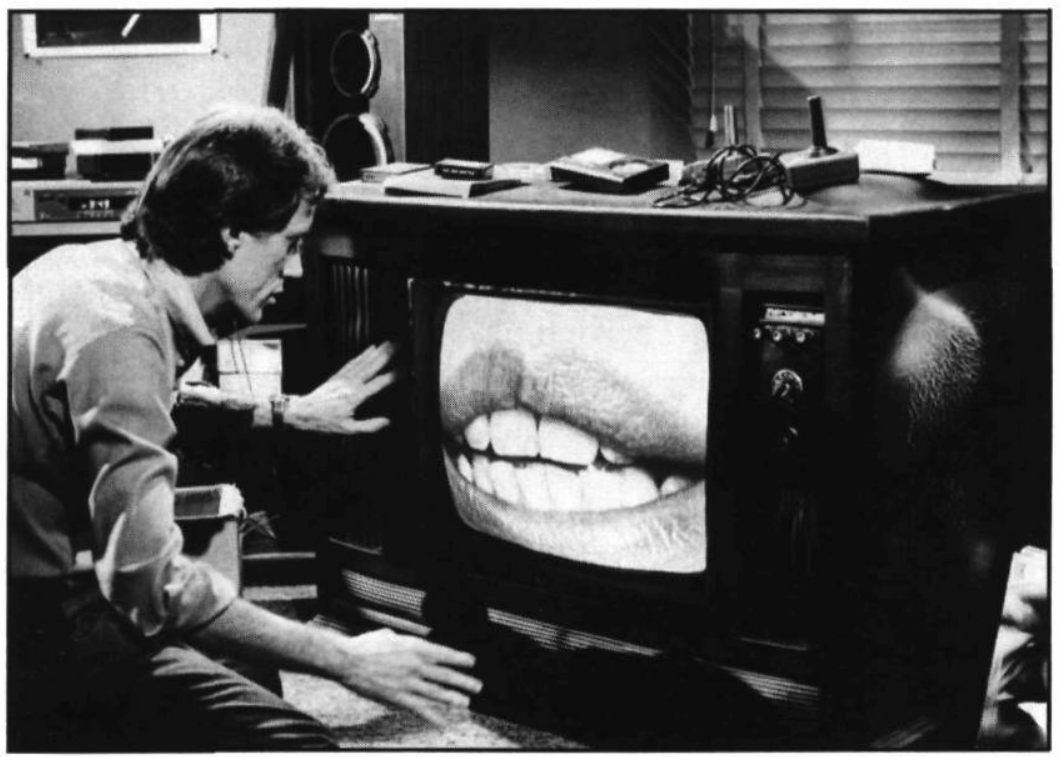

\section{Videodrome de David Cronenberg (1982)}

Collection Cinémathèque québécoise

Rappelons brièvement la trame narrative du film. Max Renn (James Woods), copropriétaire d'une petite station de télévision, est en quête d'images puissantes susceptibles d'impressionner son public et de concurrencer ainsi les grandes entreprises de l'audiovisuel. Un de ses techniciens capte par hasard le signal d'une émission provenant de toute évidence de Malaisie et qui présente une série d'images de torture sur le vif. Fasciné par ces représentations, Max les introduit dans ses jeux érotiques avec sa maîtresse Nicky Brand (Deborah Harry) pour plonger graduellement dans un climat de fascination sadique qui finit par provoquer des effets hallucinatoires sur son propre corps. Sur la piste de l'émetteur de Videodrome, il entre en contact avec un nommé $\mathrm{D}^{\prime}$ O’Blivion. Celui-ci est responsable d'un projet audiovisuel rival "à orientation religieuse " consistant à administrer des doses de rayons cathodiques de façon systématique à la population. Cependant, Max ne tarde pas à découvrir qu'il est tombé dans un piège de Spectacular Optical et qu'il a été utilisé comme cobaye pour en tester les effets sur le public. Dorénavant, son univers sera peuplé d'hallucinations. Devenu tueur à la 
solde de Videodrome, il subit des transformations constantes, physiques comme mentales et qui, brisant la fontière entre réalité et fiction, le poussent au suicide.

Il va sans dire que ce bref résumé ne rend pas compte de la complexité narrative du film. Il nous aidera, cependant, à parcourir son sens du point de vue qui nous anime, la vie des corps. D'abord, l'univers hard, mis en place dès le départ par la vision des images de torture prises sur le vif, est à l'origine du mouvement dramatique du film; lorsque Max essaie de donner un sens à ces images, il se trouve face à un homme décorporéisé, le D' O'Blivion, dont le substrat matériel se réduit au support électronique. Simple accumulation d'enregistrements vidéographiques, il ne restera de lui après son décès qu'une série inépuisable de discours prononcés devant les caméras de télévision. Ainsi un corps trop matériel, tout en demeurant image, objet de jouissance et de torture pour autrui, s'entrecroise avec un corps volatile et "intouchable», tous deux unis par un sujet porteur d'un regard fasciné. Toutes les composantes nécessaires à la naissance d'un récit sont en place. Il ne nous reste qu'à analyser de quelles manières elles s'articulent et quel type de récit en surgit.

Certes, Videodrome use d'une certaine rhétorique qui fit fureur tout au long des années soixante-dix et qui risque de le faire paraître un peu désuet à nos yeux habitués aux prouesses techniques. En effet cette production, portant sur l'univers télévisuel en pleine transformation, se fait l'écho d'un ensemble de notions qu'elle transforme en fable fantastique. Là où McLuhan évoquait l'effet de mutation perceptuelle provoquée par les nouveaux médias, Videodrome part du postulat, formulé à travers le discours du docteur, que la télévision fait partie d'ores et déjà de notre système nerveux, à tel point que le petit écran est devenu la rétine de l'œil. Littéralisation d'une métaphore, O'Blivion se réjouit de sa trouvaille formelle. Ainsi, un deuxième mythe qui a hanté la production cinématographique marginale de cette décennie est invoqué de manière explicite: le snuff, ce type de film pornographique censé se terminer par le meurtre réel des acteurs, dont les rumeurs d'existence s'accroissent entre 1975 et 1976 dans les circuits indépendants de New York ${ }^{12}$. Videodrome serait une allusion non voilée à ce modèle. 
En somme, ce double cadre d'événements qui contextualisent le film semble suggérer la possiblité de procéder à une lecture allégorique de la fiction fantastique formant le noyau de Videodrome. Et il faut bien avouer que cette démarche ne serait pas sans justification. Fredric Jameson, par exemple, en a donné une interprétation du même genre lorsqu'il a entrepris de lire les avatars politiques de la société canado-américaine contemporaine dans tous les recoins de ce cauchemar médiatique (1992, p. 2235). L'allégorie étant la figure rhétorique qui consiste à effacer le sens matériel du texte au bénéfice d'un sens figuré ou transcendental, le comportement herméneutique de Jameson ne peut réussir qu’à la condition de substituer aux événements du récit d'autres faits sociaux, politiques, bref extérieurs au texte qu'ils sont censés représenter.

Sans donner tout à fait tort à ceux qui penchent pour cette approche, notre démarche sera inverse. Aussi nous proposonsnous d'interroger le film dans sa matérialité narrative, nous refusant à traduire sa trame dans n'importe quel autre ordre, bien que le film lui-même s'y prête parfois, voire le suggère par les discours d'O'Blivion. On constatera par la suite à quel point les actions représentées s'avèrent d'une extrême richesse, autant du point de vue textuel qu'anthropologique.

\section{L'image et le regard}

Au départ, on se retrouve face à une image dépourvue autant de sens que de liaisons avec l'univers fictionnel. Max Renn est confronté à la captation d'une image quasi subliminale provenant d'une source inconnue et vraisemblablement éloignée dans l'espace: deux hommes dont les têtes sont couvertes par un capuchon fouettent une jeune fille à demi-nue qu'ils transportent près d'un mur. Ce mur fait de boue humide et électrifiée produit des décharges répétées sur son corps lui arrachant des cris de douleur. Ces trois personnages se détachent sur un décor neutre, on ne peut plus pauvre, éclairé et dominé par une tonalité rouge. La durée de cette vision n'excède pas quelques secondes et, pourtant, elle laisse des traces indélibiles chez Renn. Aucune information n'est fournie sur les personnages, aucun récit n'est amorcé, leur comportement ne favorise aucun mouvement de déduction. 
Faute d'explications, Max réagit en entrepreneur et calcule la façon de les rendre commercialement productives. Lui, qui a refusé par sa faiblesse le sexe oriental, ainsi qu'un film pornographique d'inspiration mythologique, envisage de rentabiliser sa découverte. Néanmoins, à ce motif économique vient s'ajouter une raison plus ou moins rationnelle qui nous intéresse davantage. Au-delà de sa compréhension, son œil demeure étrangement épris de cette courte vue, à peine perçue. Un œil, en somme, détaché de l'entendement, un regard ravi par son objet, prêt à le dévorer. Entre eux, aucune distance; on a affaire ici à l'émergence de la pulsion scopique.

Au fur et à mesure que les images se répètent, elles révèlent leur insistante monotonie: il n'y a ni cadre discursif, ni aucune progression vers un climax; aucun programme d'action ne peut y être décelé, aucun désir ne peut $s^{\prime} y$ installer. Les personnages ne quittent jamais la chambre, réitérant les mêmes scènes de torture, de mutilation et de meurtre. On ne peut que penser aux scènes érotiques répétées peuplant les romans du Marquis de Sade. C'est la monotonie du phantasme, le seul des mécanismes psychiques qui se dérobe à la transformation perpétuelle régissant les "formations de l'inconscient ${ }^{13}$ ". Or, ce phantasme n'appartient pas ici au personnage; il lui est plutôt imposé. Pour mieux dire, Max y assiste comme un invité. Voilà pourquoi il semble incapable de s'y installer confortablement. Et puis, une nouvelle révélation le comble d'angoisse: il n'y a aucune simulation, aucun jeu thêâtral; la scène, aussi monotone soit-elle, se déroule sur le vif, en temps réel et en l'absence du moindre trait discursif. De plus, il découvre que le signal provient de tout près, de Pittsburgh. Condition inexplicable pour le protagoniste qui se demande naïvement quel peut être l'intérêt quand la simulation en possède tous les avantages. Marsha (Lynne Gorman) formulera la réponse à l'aide de mots fort inquiétants : ces images détiennent une philosophie, et c'est cela qui les rend extrêmement dangereuses. Peu importe que le personnage plonge dans l'incompréhension, une fois que son regard s'y est accroché, ces images ne feront que le hanter, le pénétrer, lui refusant le moindre répit. 


\section{Première inscription du sujet: le phantasme}

Il reste à préciser la position que le sujet adopte à l'égard de ces images. Une première inscription se produit aussitôt et elle possède toute la force de cette machinerie de domestication de la jouissance que la psychanalyse appelle phantasme. Mais, ne nous méprenons pas, si nous venons de reconnaître un mécanisme phantasmatique dans la scène précédente, nous avons pris soin d'ajouter qu'il n'appartenait pas à Max, celui-ci demeurant hébété face au déploiement de cette violence sans raison apparente. Plus tard, en revanche, Max entreprendra une opération psychique et discursive ayant pour but de maîtriser l'angoisse provoquée par ces images. Il s'empare alors d'une cassette vidéo contenant quelques heures d'enregistrement de Videodrome et la projette chez lui en compagnie de Nicky. Considérant tout d'abord ces images fades, Nicky avoue bientôt l'excitation qu'elles éveillent en elle, révélant un penchant masochiste que des traces d'ongles sur son épaule semblent corroborer. Max luimême se laisse entraîner par les jeux érotiques de sa maîtresse. Un "travelling" avant accompagné d'une musique non diégétique nous montre, à l'arrière-plan, la télévision diffusant des scènes de torture, alors qu'au premier plan, les corps des amants s'enlacent passionnément. Mais, soudain, un "travelling" arrière accompagné d'un effet sonore provoque une dé-spatialisation de la scène, déposant le couple à l'intérieur de la salle rouge où se déroule la scène de torture. Puis, Max et Nicky disparaissent, alors engloutis par l'image. Effet délirant qui n’obéit, pourtant, à aucun point de vue subjectif et devenant par là même extrêmement inquiétant.

Il serait pertinent d'approfondir un instant la notion de phantasme pour mieux saisir son économie psychique et discursive et comprendre de façon plus précise la manière dont la scène de Videodrome est clôturée. "Scénario imaginaire, nous disent Laplanche et Pontalis, où le sujet est présent et qui figure, de façon plus ou moins déformée par les processus défensifs, l'accomplissement d'un désir et, en dernier ressort, d'un désir inconscient" (p. 152). Scène donc où deux sujets se reconnaissent dans leur imaginaire, transformant ainsi le contenu de l'action représentée. Rien d'étonnant que Freud ait conçu ces 
scènes comme des rêveries, car les voyeurs (Max et Nicky) opèrent sur l'objet de leur regard une métamorphose en tirant plaisir d'une scène de douleur et de souffrance. En d'autres termes, ils introduisent un effet pervers qui permet au désir de s'y installer. Ce n'est pas une coïncidence si Jacques-Alain Miller, dans un cadre terminologique explicitement lacanien, a défini le phantasme à l'aide du concept de jouissance: "[...] le phantasme est comme une machine à transformer la jouissance en plaisir" (p. $\left.20^{14}\right)$. Voici une perspective fort utile pour interpréter la fonction du phantasme, car celui-ci réaliserait un travail de domestication de la jouissance (extrêmement douloureuse puisque pulsionnelle), la transformant en plaisir ${ }^{15}$. Ainsi, la jouissance apparaît comme manifestation d'un versant insupportable du réel que le phantasme vient capturer et rendre plus inoffensif.

Nous sommes mieux outillés à présent pour revenir sur la scène en question. L'image de la torture, fût-elle insupportable, est transformée, suivant les voies de la perversion, en instrument de plaisir. Néanmoins, la clôture de la séquence nous suggère que l'accomplissement de cette opération n'a pas été effectif. Le phantasme se caractérisant par une conversion permanente, répétitive et stable, l'inscription de Max et Nicky ne saurait s'y adapter aisément. De surcroît, l'effet délirant souligné par la pénétration littérale des corps dans la scène phantasmatique annonce une inquiétante instabilité. Comme on le verra, le phantasme ne réussira pas à se stabiliser.

\section{Deuxième inscription : le récit}

La fragilité du phantasme, à savoir la faille ouverte entre la fascination du regard et la précarité de la scène censée protéger le sujet de la jouissance le menaçant, est la source d'une poussée métonymique qui fonde le récit. En d'autres termes, si la machine phantasmatique avait réussi à inscrire les sujets, en devenant monotone, il n'y aurait eu aucun besoin de démarrage narratif. Cependant, ici, c'est l'effondrement du phantasme qui déclenche le récit, si l'on entend par celui-ci l'itinéraire où un héros (un sujet) parcourt un univers de sens à la recherche d'une solution à une énigme ouverte, entraînant le spectateur avec lui. 
La résolution de l'énigme devrait répondre du même coup aux questions suivantes: pourquoi les premières images ont-elles secoué si profondément le sujet et pourquoi le travail du phantasme n'est-il pas parvenu à exorciser leur puissance déroutante? Un élément de réponse apparaît à travers le personnage rencontré par Max, personnage censé détenir le secret de la fiction, pris dans le réseau de la recherche audiovisuelle et connaissant les trames secrètes qui animent Videodrome. Ce personnage n'est autre que le $\mathrm{D}^{r}$ O'Blivion, incarnation d'une nouvelle fantaisie postmoderne: une image sans corps. Après avoir en vain essayé de comprendre ces images, la vision des enregistrements vidéographiques provoque chez lui des effets corporels comme des hallucinations. Dualité remarquable qui montre les limites d'une lecture allégorique, car Max subit surtout une métamorphose physique d'ordre tumoral due à une très grande consommation d'images de Videodrome.

Resté seul après la mystérieuse disparition de sa fiancée, Max ressent la hantise d'un sol qui pourrait se dérober sous lui: les objets semblent contaminés par l'érotisme humain, ils perdent leurs contours et cessent d'être inertes pour s'animer de manière inquiétante; les émissions de Videodrome, de même que celles de son ennemi O'Blivion, provoquent chez Max un comportement qui finit par devenir une programmation; Nicky ellemême s'adresse à lui de l'intérieur du petit écran, consommant le meurtre du docteur... Bref, la chronologie des événements est alors impossible à établir, l'espace se fait mobile, le réseau de faits devient inextricable. Afin d'étudier la logique hallucinatoire dans laquelle Max a plongé, on le soumet à une analyse soignée de ses cauchemars et c'est alors qu'une nouvelle scène apparaît, non plus sous ses yeux, mais totalement intériorisée: Nicky réclame son droit à être fouettée pour combler sa jouissance. Elle demeure dans la petite salle rouge qui évoque la chambre de torture du début. Max y est alors introduit, il s'empare d'un fouet et commence à frapper le poste de télévision à l'intérieur duquel on voit le corps jouissant de Nicky. Reproduction ultime de la scène phantasmatique d'origine, plénitude si l'on veut du phantasme dont l'accomplissement passe par cette sortie de la raison. Plus de regard qui s'accroche à la scène, plus de conversion de la 
jouissance en plaisir, mais plongeon dans le régime du délire, de la psychose.

Revenons sur la dimension charnelle que l'on avait mentionnée plus tôt. Devenu tueur à solde de Videodrome, le corps de Max se déchire en deux, logeant au milieu de son ventre un énorme vagin tremblant par lequel ses programmeurs vont introduire les cassettes vidéo contenant les instructions auxquelles il est censé se soumettre. Délire à implications corporelles, comme dans tous les films conçus par Cronenberg, le film instaure une matérialité qui brise le climat allégorique.

Par la suite, le récit acquerra un rythme vertigineux. Ici s'opère le choix narratif du film qui le fait plonger dans le cadre fantastique : sans les embrayeurs qui permettent de déterminer si les événements représentés répondent au point de vue subjectif de Max ou au climat surnaturel qui le domine, le caractère paranoïaque du récit échappe au protagoniste ${ }^{16}$. La trame qui se tisse ensuite n'est plus par conséquent une succession ordonnée d'événements associés les uns aux autres par un lien causal, mais bien plutôt une toile d'araignée à laquelle le héros ne peut échapper. Deux lectures deviennent alors légitimes: selon la première, nous sommes soumis à la perception délirante de Max; selon la seconde, il est devenu l'objet d'un complot criminel sous le contrôle de Videodrome qui déploie son pouvoir à l'aide d'une structure fantastique inextricable.

Le paradoxe du film réside en ce que nous ne devons pas choisir entre ces deux destinées de la fiction, mais les accepter toutes deux comme complémentaires. Voilà en fait ce qui caractérise l'aspect moderne du film. Ce n'est pas un hasard si le récit se boucle par un geste tout aussi initiatique. Nicky, à l'intérieur de l'écran, fournit à Max les instructions nécessaires pour quitter le corps qui l'enveloppe et donner naissance à la chair nouvelle. Disparition du corps, éclatement de la chair, suicide de Max, qui annonce un "rien ne va plus» de l'absence de corps dans la postmodernité.

Université de Valencia (Espagne) 


\section{NOTES}

1 Ce texte a été conçu dans le cadre du projet de recherche «Le corps humain et sa représentation audiovisuelle", subventionné par le Pla Valencià d'Investigació i Tecnologia 1996. L'auteur remercie Isabelle Labrouillère pour ses très généreuses corrections stylistiques.

2 Patrick Baudry parle à ce propos du corps extrême comme une forme d'excès liée à la dimension suicidaire (Le Corps extrême. Approche sociologique des conduites à risque, Paris: L'Harmattan, 1991).

3 Voici peut-être une des raisons de l'actualité des débats bioéthiques.

4 Ce qui revient à dire qu'elle entre dans le domaine de la névrose.

5 Et dont l'exportation rapide à d'autres pays, soi-disant sous-dévéloppés, a produit de nombreuses contradictions.

6 Ceci a été remarqué dans un domaine sociologique parallèle à celui du corps, à savoir, l'anthropologie de la mort. Voir entre autres les ouvrages de Ariès, Thomas, Illitch et Jankélévitch.

7 Silvia Tubert, dans son livre Mujeres sin sombra. Maternidad y tecnologia (Madrid: Siglo XXI, 1991), s'est occupée, dans une perspective féministe, des implications dépressives de tentatives échouées de maternité à travers les technologies de reproduction. Elle a tenté de l'expliquer à l'aide des notions lacaniennes de désir et de demande vis-à-vis de l'enfant et de la maternité. Et non pas du désir qui est tout autre chose et implique l'intervention de l'inconscient (voir la note suivante).

8 Il va sans dire que la réalisation de l'idéal est constitutivement impossible et inquiétante, car le corps auquel je midentifie (plus exactement, du point de vue psychanalytique: auquel mon Moi s'identifie) n'est qu'une image et non pas un corps réel. 9 Voici un thème qui est à la mode: la bioéthique. Une révision d'ensemble des problèmes impliqués se trouve récemment dans l'ouvrage dirigé par Paul A. Komesaroff, Troubled Bodies. Critical Perspectives on Postmodernism, Medical Ethics, and the Body (Durhan-London: Duke University Press, 1995) : état de la clinique, technologies de reproduction, conceptions de la maladie, légitimité de la recherche expérimentale, etc.

10 Nous avons entrepris cette démarche dans la troisième partie de notre dernier livre Una cultura de la fragmentatión. Pastiche, Relato y Cuerpo en el cine y la televisión (Valencia : Filmoteca Generalitat Valenciana, 1995).

11 Nous avons recours délibérément à la terminologie lacanienne qui définit ainsi le corps dans le réel par opposition au corps idéal, pur miroir imaginaire.

12 Le lecteur peut extraire quelque information sur ce sujet dans le texte, par ailleurs dérisoire, de David Kerekes et David Slater, Killing Culture. An Illustrated History of Death Film from Mondo to Snuff (Londres-San Francisco: Creation Books, 1995).

13 Voilà pourquoi Freud opposa la logique de la fantaisie (telle qu'il la nomma) aux formations de l'inconscient (symptômes, rêves, actes manqués), caractérisées par une abondante métamorphose.

14 On remarquera le fait que cette notion de jouissance constitue l'interrogation fondamentale d'un des textes freudiens les plus énigmatiques. Dans "Au-delà du principe du plaisir (1920) n, cet au-delà, cette force pulsionnelle qui cherche le mal chez le sujet, n'est autre chose que la jouissance, c'est-à-dire en termes freudiens, la pulsion de mort. On peut ainsi comprendre comment le phantasme exerce une fonction domestiquante de la jouissance et aussi, comment un effondrement de la couverture fantasmatique fait surgir l'angoisse.

15 C'est ainsi que Jacques-Alain Miller décrit le travail du phantasme, tout en affirmant, dans une perspective lacanienne, le caractère sauvage et indomptable de la

Entre le corps évanescent et le corps supplicié: Videodrome et les fantaisies postmodernes 
jouissance, car elle se situe "au-delà du principe du plaisir " ( Dos dimensiones clínicas: síntoma y fantasma ", Analytica, Buenos Aires: Navarin, 1983, p. 9-69).

16 Ce qui est une caractéristique générale de l'économie moderne à la différence de l'énonciation du film classique hollywoodien où, quelle que fût sa complexité narrative, tous les embrayeurs étaient en marche ou se reconstituaient à la fin du film.

\section{OUVRAGES CITÉS}

Ariès, Philippe. L'Homme devant la mort. Paris: Seuil, 1977.

Baudry, Patrick. Le Corps extrême. Approche sociologique des conduites à risque. Paris: L'Harmattan, 1991.

Foucault, Michel. Naissance de la clinique. Une archéologie du regard médical. Paris: P. U F. / Quadrige, 1990.

Foucault, Michel. Histoire de la sexualité. I. La volonté de savoir. Paris: Gallimard, 1976.

Foucault, Michel. Surveiller et punir. Naissance de la prison. Paris: Gallimard, 1975.

Freud, Sigmund. "Au-delà du principe du plaisir (1920)", Essais de psychanalyse. Paris: Payot, 1975, p. 7-81.

Illich, Ivan. Némésis médicale. L'expropriation de la santé. Paris: Seuil, 1975.

Jameson, Fredric. The Geopolitical Aesthetic. Cinema and Space in the World System. London-Indianapolis: B. F. I. \& University of Indiana Press, 1992.

Jankélévitch, Vladimir. La Mort. Paris: Flammarion, 1977.

Kerekes, David et Slater, David. Killing for Culture. An Illustrated History of Death Film from Mondo to Snuff. Londres-San Francisco : Creation Books, 1995.

Komesaroff, Paul A. (direction). Troubled Bodies. Critical perspectives on Postmodernism, Medical Ethics, and the Body. Durhan-London: Duke University Press, 1995.

Lacan, Jacques. Le Séminaire. Livre I. Les écrits techniques de Freud. Paris: Seuil, 1975.

Laplanche, Jean et Pontalis, Jean-Bertrand. Vocabulaire de la psychanalyse. Paris: P. U. F., 1984.

Miller, Jacques-Alain. "Dos dimensiones clínicas: síntoma y fantasma ». Analytica. Buenos Aires: Navarin, 1983, p. 9-69.

Sánchez-Biosca, Vicente. Una cultura de la fragmentatión. Pastiche, Relato y Cuerpo en el cine y la televisión. Valencia: Filmoteca Generalitat Valenciana, 1995.

Thomas, Louis-Vincent. La Mort en question. Traces de mort, mort des traces. Paris: L'Harmattan, 1991.

Thomas, Louis-Vincent. Rites de mort. Pour la paix des vivants. Paris: Fayard, 1985.

Tubert, Silvia. Mujeres sin sombra. Maternidad y tecnologia. Madrid: Siglo XXI, 1991. 